\title{
How to Develop Gluten-Free Foods for a Healthy Gut
}

\author{
Federico Morreale, Nicoletta Pellegrini* \\ Department of Food Science, Human Nutrition Unit, University of Parma, Parma, Italy \\ *Corresponding author: nicoletta.pellegrini@unipr.it
}

Received April 05, 2015; Revised May 05, 2015; Accepted June 05, 2015

Keywords: celiac disease, gut inflammation, gluten-free foods, gluten-free diet, gut barrier, gut microbiota

Cite This Article: Federico Morreale, and Nicoletta Pellegrini, "How to Develop Gluten-Free Foods for a Healthy Gut.” International Journal of Celiac Disease, vol. 3, no. 2 (2015): 44-47. doi: 10.12691/ijcd-3-2-10.

Celiac disease (CD) is a chronic systemic autoimmune disorder sustained by an inappropriate response to gluten. The term gluten is often used to encompass the prolamins, specific storage ethanol-soluble proteins, of wheat, rye and barley at the base of the characteristic immune response of celiac disease. The development of clinical symptoms of $\mathrm{CD}$ is a consequence of the encounter between an environmental trigger and a genetically predisposed host, with the possible participation of other environmental cofactors like drugs, intestinal infections or infant-feeding practices. Genetically susceptible individuals develop an autoimmune injury to the gut, skin, joints, liver, brain, heart, uterus and other organs [1].

There are at least 50 toxic epitopes in gluten peptides exerting citotoxic, immunomodulatory and gut-permeating activities, but most of them are digested by gastric, pancreatic and intestinal proteases [2]. However, in celiac patients, one of these epitopes promotes the release of zonulin peptide in small bowel mucosa. Zonulin activates an intracellular pathway that ends with the disassembly of the tight junction (TJ). This directly affects the opening of the paracellular pathway, allowing the toxic 33-mer gliadin peptide to make contact with the tissue transglutaminase and the antigen-presenting cells in the lamina propria [3] generating the immune response.

CD may affect as many as $1 \%$ to $3 \%$ of the European and North American population [1]. The CD prevalence is significantly higher than that recognized 20 years ago; in fact, recent studies have demonstrated that the incidence is increasing in North America and Europe [4]. A change in human genetics is unlikely, because it would take too long even in the event that there was an environmental variable that exerts a selective pressure. So the high and increasing incidence may partly be explained by high physician awareness, large-scale efforts to screen at-risk groups (such as women and patients with other autoimmune diseases) and the introduction of screening diagnostics more complete and accurate, such as the anti-endomysial antibody and anti-tissue transglutaminase antibody serological assay. Ludvigsson et al. [5], in a recent study on the US population, proposed an environmental exposure cause for the increased incidence of CD in US, i.e. the increased consumption, in frequency and amount, of gluten in foods available for the US population. However, the lack of reliable data on the amount of gluten in foods and of data from other countries does not allow the reaching of a conclusion.

Besides CD and wheat allergy, there are other cases of gluten reactions in which neither allergic nor autoimmune mechanisms are involved. These are generally defined as gluten sensitivity (GS) or non celiac gluten sensitivity (NCGS), a condition in which symptoms are probably triggered by gluten ingestion, in the absence of celiacspecific antibodies and of classical celiac villous atrophy, with variable human leukocyte antigen status and a variable presence of first generation anti-gliadin antibodies [6].

Since the ingestion of gluten is correlated with an inflammatory prolonged condition and, in turn, results in a malabsorption of essential micronutrients, such as iron, zinc, calcium, folic acid and fat-soluble vitamins, the only known therapy is a lifelong gluten-free diet (GFD) that can lead to a mucosal healing and an improvement in pathological symptoms and nutritional status. The GFD excludes any products derived from wheat, barley and rye grains [7]. Not considering the particular, and not very frequent, case of the non responsive celiac patients, the elimination of gluten usually induces the clinical improvement within days or weeks, even though the histological recovery takes months or even years, especially in adults, in whom the mucosal recovery may also be incomplete [8].

Lebwohl et al [9] reported that the gut injury is correlated with the prevalence of villous atrophy in adulthood and is influenced by the prolonged course of symptoms prior to diagnosis, the discontinuous follow-up biopsy and a late institution of GFD. Adults and older people may have a longer and untreated CD than younger and, as a consequence, their gut inflammation and atrophy may be more persistent [9]. The persistent pro-inflammatory stimuli, related to the prolonged presence of proinflammatory mediators of the innate immune system and the prevalence of Th1 cells of adaptive immune system, result in a gradual overload of the gut with reactive oxidant species that gradually weaken the antioxidative defense system [10]. Therefore, such continued proinflammatory stimuli to the intestinal mucosa result in: i) a tissue hardly damaged, with a loss of barrier function and compartmentalization; ii) a reduction of mucus layer and its protective and regulatory functions; iii) an altered 
exposure and tolerance to sequestered antigens, autoantigenes or components of microbiota.

The identification of intestinal microbiota as a prominent environmental factor shaping diverse aspects of the intestinal and extra-intestinal health and diseases has fueled an intense interest in defining the mechanisms underlying host-microbiota interactions [11]. It is well known that the human gastrointestinal tract is a complex and dynamic environment sheltered by a vast number and variety of commensal microorganisms. This balanced microecosystem provides the host a natural defense against the invasion of potential pathogens. Studies of the role of the gut microbiota in CD pathophysiology are still in their infancy and to date it is still unclear whether an altered microbiota in CD patients could be the cause or the consequence of the pathology. De Sousa Moraes et al. [12] recently reviewed the main findings on this issue.

It is hypothesized that the proportion of gram-negative and gram-positive bacteria may be of importance in CD patients and that gram-negative bacteria in genetically susceptible individuals may contribute to the loss of tolerance to gluten. Low levels of Lactobacillus and Bifidobacterium species in CD children have been observed in favor to a major prevalence of proinflammatory gram-negative bacteria, such as Bacterioides-Prevotella and Escherichia coli [12]. In addition, Bernardo et al [13] have recently suggested that in the duodenum of celiac patients with respect to healthy controls, independently of interleukin 15 (IL-15) levels or of the inflammatory intestinal condition, there is a high expression of the IL15-receptor-alpha. Such high expression could determine a lower threshold of activation of innate immune response in CD predisposed individuals with respect to healthy subjects. As a consequence, it would be speculated that these individuals could be more sensitive to the environmental triggers, such as microbiota fragments and metabolites, than healthy ones.

Considering all the cited studies concerning the intestinal dysbiosis [12] and the possible constitutive alteration of the innate immune response [13], it might be possible that the celiac patient could have alterations that may be the basis of inflammatory disorders untreatable with just the elimination of gluten from the diet. Reducing the inflammatory stress could allow the intestinal tissue to recover its barrier function between intestinal lumen and submucosal space, thus preventing the food antigens to reach the effector cells of the adaptive immune system. These suggestions should be considered in the development of gluten-free (GF) products. To date, the composition of GF products has been formulated with the major purpose of eliminating the gluten and overcoming the technological gap resulting from its elimination. Therefore, in the future, such products should also be formulated, for instance, for controlling the intestinal dysbiosis and for promoting the balance of intestinal microbiota towards gram-positive species and, in turn, the inflammatory condition.

For a variety of reasons, the market for GF products is in a continuous expansion. This huge requirement determines an increasing demand for GF products with sensory and nutritive characteristics suitable for replacing, at least partially, the traditional wheat-based products [14].

From a technological point of view, gluten is a structure-building protein, which contributes to the formation of a strong protein network. This confers viscoelasticity to the dough and allows the wheat flour to be processed into a wide range of products. As a consequence, the technological process of GF cereal based products entails some difficulties. For example, the GF batter and dough have been described as being less cohesive and elastic than wheat dough, more difficult to handle and as having poor gas retention. GF yeast-raised baked goods have been portrayed as having a low volume, a pale crust, a crumbly texture and other problems that affect the taste and acceptability by the consumer [15]. Therefore, obtaining high-quality yeast-raised baked goods is a technological challenge that leads to the search for ingredients, additives and technologies that can improve the bread-making performance of GF flour. The techniques and the recipes for improving the sensory quality of the GF products have been recently reviewed [14].

From the nutritional point of view, the exclusion of gluten does not entail particular problems, being a mixture of proteins with a low nutritional and biological value. However, its replacement affects the other nutrients in GF products, especially in the bread category, such foods being poorer in proteins, but richer in total and saturated fats when compared with their gluten-containing counterparts [16]. In pasta, flours and cereal bars, there are some additional nutritional concerns, such as high levels of sodium and cholesterol [16]. A recent review from Pellegrini and Agostoni [17] also highlights the fact that such goods may contain less micro-nutrients with respect to the gluten-containing counterparts. Lately, in some GF recipes the use of pseudo-cereals and non-conventional flours and starches as ingredients to enrich the dough in micro-nutrients and fiber has been reported [18]. This choice is also due to the market demand arising from the consumer's request for nutritional ingredients in GF products.

A celiac patient, in the context of gluten exclusion, should have dietary habits in line with the principles of the Mediterranean diet, a healthy and protective food model, or one with other dietary regimes promoted by the national and international health organizations. A healthy dietary pattern is rich in plant foods, such as cereals, fruit, vegetables, legumes, tree nuts, seeds and olives, with a moderate intake of fish and seafood, eggs and dairy products and a low consumption of red meat and other processed meats. This pattern ensures an adequate intake of macro- and micro-nutrients and bioactive molecules that promote health in a general population. However, the widespread adherence to the western lifestyle contributes to the custom of using convenience foods and meals.

Calder and colleagues [10] in a work commissioned by ILSI Europe on the inflammatory disease process and the interaction with nutrition discussed the connection between the western lifestyle (urban life, pollution, psychological stress, infections, reduced physical activity and dietary habits poor in fruit and vegetables and rich in nutrient-poor foods containing high levels of sugar and saturated fats) and the resulting oxidative stress in the body. Moreover, the authors highlighted the negative effect that this lifestyle may have on the gut barrier of the entire population and the severe implications for people who suffer from chronic intestinal diseases, like celiac disease. However, the eating of such products is often a 
choice dictated both by their convenient accessibility and the strong desire of the celiac patient for social acceptance [19]. Theoretically, to follow a strict GFD is simple, but its management is much more difficult. It requires wellmotivated patients, supported by an experienced dietitian, family and community.

Today a celiac patient is prone to include in his or her diet industrial GF foods, like bread, pasta, cookies, cereal bars, pizza or prepared meals. The major concern about those foods is that the food industry still employs primarily refined flours and starches from maize and rice, which are poor in micro-nutrients, as conventional substitutes for wheat flour and it enhances the taste of them by the addition of sodium and fats. Nowadays, there are some nutrients, such as polyunsaturated fatty acids, antioxidant vitamins, polyphenols, prebiotics and probiotics, to which the nutritional research is paying special attention, exploring also their effect on immune/inflammatory markers. In developing GF products, the food industry should take into account such research.

An aspect that is becoming extremely relevant is the study of the gut barrier integrity and the right balance of the gut microbiota. In fact, the question of how we can improve the gut barrier function and the gut microbiota, not only for healthy people but also for celiac patients, has assumed a key role for maintaining human health. The research on the modulation of gut barrier integrity and permeability by the diet is recently starting and there are some dietary factors that have been proposed to improve such aspects [20]. Among them, two major factors could be identified: i) nutrients/prebiotics; ii) probiotics [20].

Some nutrients and non-nutrients might act in an antiinflammatory manner by reinforcing the regulatory pathways that control the inflammatory responses. Polyphenols, prebiotic food components or probiotic bacteria might be of great interest as future "therapy" of the intestinal barrier-related diseases [20,21]. In this way, tailored developed foods might contribute to the robustness of homeostatic control and help to reduce the risk that the inflammatory response, acute or chronic, determines a continuous damage to the gut [10].

Recently, some studies on the gut of the celiac patients focused on the balance and the composition of the intestinal microorganisms and also on the negative effects of dysbiosis [22,23] as a consequence of the GFD [12,24]. However, to date, there is a lack of consensus about the exact bacterial composition in patients with CD [12]. Nevertheless, as introduced above, the importance has been demonstrated, also for the celiac patients, of the presence of Lactobacillus and Bifidobacterium, grampositive and anti-inflammatory species, to improve the integrity of gut trans-epithelial barrier by i) contrasting the colonization, for example, by Escherichia coli or Shigella, considered as the pro-inflammatory bacteria, and ii) reducing the release of pro-inflammatory cytokines by the activated monocytes [12]. However, in the perspective of positively modulating the gut microbiota of celiac patients, the supplementation of preparations containing probiotics currently does not meet the consensus of the scientific community and, moreover, the GF foods are not goods traditionally functionalized with probiotics. A more concrete way for obtaining a correct microorganism balance in favor of gram-positive anti-inflammatory species should be the introduction of resistant polysaccharides from plant foods and the reduction of fatty acids in GF foods [25].

An aid to reduce the use of fat ingredients rich in saturated fatty acids in breads comes from an ancient production technique: sourdough fermentation. Sourdough is a mixture of lactic acid bacteria (LAB), yeast and flour. This technique, applicable to various combinations of flour, positively influences all aspects of bread quality: texture, aroma, nutritional properties and shelf life. Also particularly interesting is the production of a wide variety of long chain sugar polymers, called exopolysaccharides (EPS), from sucrose during the sourdough fermentation by many LAB. Some EPS can improve the GF bread technological quality by acting as hydrocolloids and potentially its nutritional quality by acting as prebiotics and anti-inflammatory and immunomodulatory agents [26]. Moreover, due to the presence of such compounds, substituting the classic guar gum or hydroxypropyl metilcellulose, the GF breads could be perceived as more natural by consumers [18].

EPS combined with alternative flours, resistant starch (RS) and viscose fibers are also studied for their role in the glycemic response to GF bakery foods [26]. Although these studies need to be deepened, the GF breads with sourdough could also be the answer to the demand for clean labels, natural products and a reduced use of additives [14].

EPS, RS and resistant non starch polysaccharides, usually defined prebiotics, are considered simpler and more effective modulators of the gut microbiota with respect to probiotics [27]. These substances, which occur naturally in foods or are extracted from natural sources or synthesized and added to processed foods, are not, or are minimally digested, in the small intestine. They are carried on into the large intestine where they are fermented selectively from the beneficial bacterial groups, such as Bifidobacterium, Lactobacillus and Eubacterium species. The metabolites that those beneficial species produce can be used as energy source, immune system enhancers or facilitators of mineral uptake. In particular, among the most represented metabolites, there are short-chain fatty acids (SCFAs), such as acetate, propionate, butyrate and valerate. These acids can disrupt both the vitality and gene expression of pro-inflammatory and pathogenic species or, as in the case of butyrate, they could play a particular role for maintaining the intestinal barrier integrity by acting on the TJ [28].

Despite the fact that studies conducted to date concern mainly the role of prebiotics in the colon, bacterial species, such as Bifidobacterium, Lactobacillus and Eubacterium, are also present, though in small amounts, in the small intestine. Therefore, it would be reasonable to think that foods containing prebiotics might also affect the balance of resident microbiota in the small intestine. This consideration is supported by the presence of SCFAs in the small intestine [29].

Food industries already use dietary fibers as ingredients in the formulation of GF food, although, at present, dietary fibers are mainly used for their technological properties, instead of as prebiotics. Therefore, these ingredients or the sourdough metabolites (e.g., EPS) should be intentionally used in the future formulation of GF foods. 
Although the studies are in their infancy, a microbiota imbalance and a pro-inflammatory status in the gut are assumable conditions in CD patients, even though on GFD. Therefore, in the development of GF foods the goal of achieving a healthy gut should be pursued. However, further studies are needed to better understand the role of prebiotics in the modulation of gut microbiota. Moreover, the studies regarding the role of the dietary habits of CD patients on the gut microbiota and the effect of dysbiosis in modulating the inflammatory stress in the celiac patient intestine should be extended.

\section{References}

[1] Zanoni, G., Navone, R., Lunardi, C., Tridente, G., Bason, C., Sivori, S., Beri, R., Dolcino, M., Valletta, E., Corrocher, R., Puccetti, A., "In celiac disease, a subset of autoantibodies against transglutaminase binds toll-like receptor 4 and induces activation of monocytes," PLoS Medicine, 3(9), 1637-1653. Sep.2006.

[2] Barone, M., Troncone, R., Auricchio, S., "Gliadin peptides as triggers of the proliferative and stress/innate immune response of the celiac small intestinal mucosa," International Journal of Molecular Sciences, 15(11), 20518-20537. Nov. 2014.

[3] Fasano, A., "Leaky gut and autoimmune diseases," Clinical Reviews in Allergy and Immunology, 42(1), 71-78. Nov.2012.

[4] World Gastroenterology Organisation - WGO, "Celiac Disease World Gastroenterology Organisation Global Guidelines," Apr.2012.

[5] Ludvigsson, J.F., Rubio-Tapia, A., van Dyke, C.T., Melton, L.J., Zinsmeister, A.R., Lahr, B.D., Murray, J.A, "Increasing incidence of celiac disease in a North American population," The American Journal of Gastroenterology, 108(5), 818-24. Mar.2013.

[6] Catassi, C., Bai, J.C., Bonaz, B., Bouma, G., Calabrò, A., Carroccio, A., Castillejo, G., Ciacci, C., Cristofori, F., Dolinsek, J., Francavilla, R., Elli, L., Green, P., Holtmeier, W., Koehler, P., Koletzko, S., Meinhold, C., Sanders, D., Schumann, M., Schuppan, D., Ullrich, R., Vécsei, A., Volta, U., Zevallos, V., Sapone, A., Fasano, A., "Non-celiac gluten sensitivity: The New Frontier of Gluten Related Disorders," Nutrients, 5(10), 3839-3853. Sep.2013.

[7] Galli, G., Esposito, G., Lahner, E., Pilozzi, E., Corleto, V.D., Di Giulio, E., Aloe Spiriti, M.A., Annibale, B., "Histological recovery and gluten-free diet adherence: a prospective 1-year follow-up study of adult patients with coeliac disease," Alimentary Pharmacology \& Therapeutics, 40(6), 639-647. Jun.2014.

[8] Green, P.H.R., Cellier, C., "Celiac disease," The New England Journal of Medicine Review, 3571731-43. Oct.2007.

[9] Lebwohl, B., Murray, J.A., Rubio-Tapia, A., Green, P.H.R., Ludvigsson, J.F, "Predictors of persistent villous atrophy in coeliac disease: a population-based study," Alimentary Pharmacology and Therapeutics, 39(5), 488-495. Mar.2014.

[10] Calder, P.C., Albers, R., Antoine, J-M., Blum, S., Bourdet-Sicard, R., Ferns, G.A., Folkerts, G., Friedmann, P.S., Frost, G.S., Guarner, F., Løvik, M., Macfarlane, S., Meyer, P.D., M’Rabet, L., Serafini, M., van Eden, W., van Loo, J., Vas Dias, W., Vidry, S., Winklhofer-Roob, B.M., Zhao, J., "Inflammatory disease processes and interactions with nutrition," The British Journal of Nutrition, 101 SupplS1-S45. May.2009.

[11] Sommer, F., Bäckhed, F., "The gut microbiota-masters of host development and physiology," Nature Reviews Microbiology, 11(4), 227-38. Apr.2013.

[12] De Sousa Moraes, L.F., Grzeskowiak, L.M., de Sales Teixeira, T.F., do Carmo Gouveia Peluzio, M., "Intestinal microbiota and probiotics in celiac disease," Clinical Microbiology Reviews, 27(3), 482-489. Jul.2014.
[13] Bernardo, D., Garrote, J.A., Allegretti, Y., León, A., Gómez, E., Bermejo-Martin, J.F., Calvo, C., Riestra, S., Fernández-Salazar, L., Blanco-Quirós, A., Chirdo, F., Arranz, E., "Higher constitutive IL15R $\alpha$ expression and lower IL-15 response threshold in coeliac disease patients," Clinical and Experimental Immunology, 154(1), 64-73. Jun.2008.

[14] Capriles, V.D., Arêas, J.A.G., "Novel approaches in gluten-free breadmaking: Interface between food science, nutrition, and health," Comprehensive Reviews in Food Science and Food Safety, 13(5), 871-890. Apr.2014.

[15] O’Shea, N., Arendt, E., Gallagher, E., "State of the art in glutenfree research," Journal of Food Science, 79(6), 1067-1076. Mar.2014.

[16] Miranda, J., Lasa, A., Bustamante, M.A., Churruca, I., Simon, E., "Nutritional differences between a gluten-free diet and a diet containing equivalent products with gluten," Plant Foods for Human Nutrition, 69(2), 182-187. Mar.2014.

[17] Pellegrini, N., Agostoni, C., "Nutritional aspects of gluten-free products," Journal of the Science of Food and Agriculture. Jan.2015.

[18] Matos, M.E., Rosell, C.M., "Understanding gluten-free dough for reaching breads with physical quality and nutritional balance," Journal of the Science of Food and Agriculture, 95(4), 649-860. May.2014.

[19] Rose, C., Howard, R., "Living with coeliac disease: a grounded theory study," Journal of Human Nutrition and Dietetics, 27(1), 30-40. Feb.2014.

[20] Bischoff, S.C., Barbara, G., Buurman, W., Ockhuizen, T., Schulzke, J-D., Serino, M., Tilg, H., Watson, A., Wells, J.M., "Intestinal permeability - a new target for disease prevention and therapy," BMC Gastroenterology, 14(1), 189. Nov.2014.

[21] Duda-Chodak, A., Tarko, T., Satora, P., Sroka, P., "Interaction of dietary compounds, especially polyphenols, with the intestinal microbiota: a review," European Journal of Nutrition, 54(3), 325341. Feb.2015.

[22] Wacklin, P., Kaukinen, K., Tuovinen, E., Collin, P., Lindfors, K., Partanen, J., Mäki, M., Mättö, J., "The duodenal microbiota composition of adult celiac disease patients is associated with the clinical manifestation of the disease," Inflammatory Bowel Diseases, 19(5), 934-41. Apr.2013.

[23] Ducatelle, R., Eeckhaut, V., Haesebrouck, F., Van Immerseel, F., "A review on prebiotics and probiotics for the control of dysbiosis: present status and future perspectives," Animal, 9(01), 43-48. Aug. 2014.

[24] Sanz, Y., Sánchez, E., Marzotto, M., Calabuig, M., Torriani, S., Dellaglio, F., "Differences in faecal bacterial communities in coeliac and healthy children as detected by PCR and denaturing gradient gel electrophoresis," FEMS Immunology and Medical Microbiology, 51(3), 562-568. Nov.2007.

[25] Salonen, A., de Vos, W.M., "Impact of diet on human intestinal microbiota and health," Annual Review of Food Science and Technology, 5239-62. Jan.2014.

[26] Moroni, A.V., Dal Bello, F., Arendt, E.K., "Sourdough in glutenfree bread-making: An ancient technology to solve a novel issue?," Food Microbiology, 26(7), 676-684. Jul.2009.

[27] Di Bartolomeo, F., Startek, J.B., Van Den Ende, W., "Prebiotics to fight diseases: Reality or fiction?," Phytotherapy Research, 27(10), 1457-1473. Dec.2013.

[28] Plöger, S., Stumpff, F., Penner, G.B., Schulzke, J.D., Gäbel, G., Martens, H., Shen, Z., Günzel, D., Aschenbach, J.R., "Microbial butyrate and its role for barrier function in the gastrointestinal tract," Annals of the New York Academy of Sciences, 1258(1), 52-59. Jul.2012.

[29] Gantois, I., Ducatelle, R., Pasmans, F., Haesebrouck, F., Hautefort, I., Thompson, A., Hinton, J.C., Van Immerseel, F., "Butyrate specifically down-regulates salmonella pathogenicity island 1 gene expression," Applied and Environmental Microbiology, 72(1), 946-949. Jan.2006. 